\title{
RANBP9 suppresses tumor proliferation in colorectal cancer
}

\author{
CHUNZHI QIN, QIN ZHANG and GUANGBIN WU \\ Department of General Surgery, Jinshan Hospital, Fudan University, Shanghai 201508, P.R. China
}

Received August 8, 2018; Accepted February 11, 2019

DOI: $10.3892 /$ ol.2019.10134

\begin{abstract}
RAN binding protein 9 (RANBP9) is widely expressed in mammalian tissues, including osteosarcoma, lung, gastric and breast cancer tissues. However, currently, not much is known about the role of RANBP9 in colorectal cancer (CRC). In the present study, RANBP9 expression in $\mathrm{CRC}$ tissues and cell lines was measured by immunohistochemistry and western blotting, respectively. Subsequently, $R A N B P 9$-short hairpin RNA (shRNA) and RANBP9 plasmids were constructed and transfected into HCT116 and HT29 cells. The effects of RANBP9 knockdown were assessed by Cell Counting kit- 8 and colony formation assays, and its effects on tumorigenicity in a nude mouse animal model were investigated. The effect of RANBP9-shRNA on cell cycle progression was analyzed by flow cytometry, while cell cycle-associated protein expression levels were examined by western blotting. Compared with in paired normal mucosa, RANBP9 was overexpressed in CRC tissues. Inhibition of RANBP9 in HCT116 and HT29 cells significantly promoted cell growth, colony formation and $\mathrm{S}$ phase transition, and increased tumorigenesis in vivo. Accordingly, RANBP9 overexpression inhibited cell growth and colony formation. Knockdown of RANBP9 was associated with upregulated cyclin A2 in the two cell lines. In conclusion, RANBP9 served an inhibitory role in CRC in vitro and in vivo. Therefore, RANBP9 may be considered a potential target for treatment of CRC.
\end{abstract}

\section{Introduction}

Colorectal cancer (CRC) is the third most common cancer worldwide. Its prognosis is poor and the total 5-year survival rate is $65 \%$ (1). CRC is considered to evolve from conventional adenoma, which gradually accumulates genetic or epigenetic changes, which eventually lead to cancer. The most common and characteristic genetic alterations occur in the APC regulator of WNT signaling pathway, tumor protein p53, KRAS proto-oncogene GTPase and mismatch repair genes (2).

Correspondence to: Dr Guangbin Wu, Department of General Surgery, Jinshan Hospital, Fudan University, 1508 Longhang Road, Shanghai 201508, P.R. China

E-mail: jackvv@163.com

Key words: RAN binding protein 9, cell cycle, colorectal cancer
RAN binding protein 9 (RANBP9) is a 90-kDa protein, which was initially screened and identified by Nishimoto et al in a yeast two-hybrid experiment (3). RANBP9 is conserved in various organisms, including humans, rhesus monkeys, mice and frogs (4,5). RANBP9 is widely expressed in mammalian tissues and is distributed in the nucleus and cytoplasm (5); however, its biological functions remain unclear. RANBP9 overexpression can reduce dendritic arbor and spine density, and can accelerate loss of dendritic spines in an Alzheimer's disease mouse model (6,7). Furthermore, RANBP9 has been demonstrated to be involved in the nucleation of the central microtubule, affecting cell division and differentiation (8). Additionally, RANBP9 has been suggested as a platform for the interaction of cell signaling molecules, including cell surface receptors, nuclear receptors, transcription factors and cytoplasmic kinases $(4,9)$. Similar to the majority of RAN binding proteins, RANBP9 is functionally associated with the $\beta$-importin receptor family, which is responsible for transporting proteins into the nucleus (8). Additionally, RANBP9 has been associated with osteosarcoma, lung, gastric and breast cancer (10-14); however, its systematic effects in cancer remain to be investigated.

In the present study, overexpression of RANBP9 in CRC was identified. Additionally, its suppression by short hairpin RNA (shRNA) promoted cell proliferation and transition from $\mathrm{S}$ phase. Furthermore, cyclin A2 expression was demonstrated to be associated with RANBP9 knockdown. In conclusion, the findings of the present study suggested that RANBP9 may be a potent anti-oncogene in CRC.

\section{Materials and methods}

Clinical specimens and immunohistochemistry (IHC) scoring. A total of 75 consecutive specimens (tumors and paired normal tissues) from $53(70.7 \%)$ male patients and 22 (29.3\%) female patients (median, 65 years; range, 32-81) with CRC who underwent radical colectomy were collected at the Department of General Surgery, Jinshan Hospital, Fudan University (Shanghai, China) between January and June 2012. IHC was performed and investigated as described previously (15). In January 2018, 12 fresh specimens (tumors and paired normal tissues) from patients with CRC were randomly collected from the same hospital for detection of RANBP9 expression using western blotting (WB). The age range was 36-79 years (median, 56 years), including $9(75.0 \%)$ male patients and 3 (25.0\%) female patients. Ethical approval was obtained from the Clinical Research Ethics Committee of Jinshan Hospital, 
Fudan University. Written informed consent for the acquisition and use of tissue samples was obtained from all patients.

Cell culture. The CRC cell lines HCT116, HT29, SW480, SW620, RKO, Lovo, Caco2 and DLD1 were obtained from Nanjing KeyGen Biotech Co., Ltd. (Nanjing, China). HCT116 and HT29 cells were maintained in McCoy's 5A medium (Nanjing KeyGen Biotech Co., Ltd.), while the other cell lines were cultured in Dulbecco's modified Eagle's medium (HyClone; GE Healthcare Life Sciences, Logan, UT, USA). The media were supplemented with $10 \%$ fetal bovine serum (Gibco; Thermo Fisher Scientific, Inc., Waltham, MA, USA) and cultured in a humidified atmosphere containing $5 \% \mathrm{CO}_{2} / 95 \%$ air at $37^{\circ} \mathrm{C}$.

Plasmids and cell transfection. RANBP9-shRNA (NM_005493.2) targeting sequence (5'-GGAATTGGATCC TGCGCAT-3') was designed and constructed. Non-sense sequence (5'-TTCTCCGAACGTGTCACGT-3') was used as a control-shRNA. shRNA sequences were then cloned into GV112 plasmids (Shanghai GeneChem Co., Ltd., Shanghai, China), which were packed into a lentivirus using 293T cells, and the lentivirus was harvested and purified by Shanghai GeneChem Co., Ltd. HCT116 and HT29 cells at a density of $10^{4}$ cells per well in 6-well plates were infected with the lentiviruses at 20 multiplicity of infection. The stably infected cells were enriched by puromycin $(2 \mu \mathrm{g} / \mathrm{ml}$; Sigma-Aldrich; Merck KGaA, Darmstadt, Germany). The time interval between infection and subsequent experimentation was $>1$ month. To induce overexpression of RANBP9, HCT116 and HT29 cells $\left(1 \times 10^{6}\right)$ were transfected with $2 \mu \mathrm{g} / \mathrm{ml} \mathrm{GV} 141-R A N B P 9$ or empty vectors (Shanghai GeneChem Co., Ltd.) using FuGENE HD transfection reagent (Promega Corporation, Madison, WI, USA). After $72 \mathrm{~h}$ of transfection, WB analysis was conducted to verify RANBP9 expression with a RANBP9 antibody. In the shRNA experiment, HCT116 or HT29 cells were infected with the empty vector in the blank control group.

Cell Counting kit-8 (CCK-8) assay. HCT116 and HT29 cells $(4,000$ cells/well) were seeded into 96 -well plates. Cell viability was measured using a CCK-8 assay (Dojindo Molecular Technologies, Inc., Kumamoto, Japan) at several time points over 3 days. Briefly, cells were incubated with $10 \mu \mathrm{l} \mathrm{CCK}-8$ for $1 \mathrm{~h}$ at $37^{\circ} \mathrm{C}$. Subsequently, the optical density was detected at $450 \mathrm{~nm}$ using a multifunctional plate reader (BioTek Instruments, Inc., Winooski, VT, USA) according to the manufacturer's protocol.

Anchorage-independent colony formation assay. Complete medium with $0.5 \%$ agarose was layered in a 6 -well plate and placed at room temperature to concretion. HCT116 and HT29 cells (200/well) were inoculated into the plate. Matrigel ( $250 \mu \mathrm{g} / \mathrm{ml}$; BD Biosciences, San Jose, CA, USA) was mixed with the cell solutions for $1 \mathrm{~min}$ in advance. When the number of cells in the majority of the single colonies were $>50$, the cells were stained with $0.005 \%$ crystal violet (Biosharp, Hefei, Anhui, China) for $1 \mathrm{~h}$ at room temperature. Subsequently, the number of visible colonies was counted.

Flow cytometry (FCM). HCT116 and HT29 cells were harvested and prepared as cell suspensions. Adherent cells were digested with EDTA-free trypsin and washed twice with ice-cold PBS. The cells were subsequently fixed with $70 \%$ ethanol at $-20^{\circ} \mathrm{C}$ for $2 \mathrm{~h}$, and stained with PI/RNase staining solution (BD Biosciences) for $1 \mathrm{~h}$ at $4^{\circ} \mathrm{C}$. Following incubation with PI for $15 \mathrm{~min}$ at room temperature in the dark, the cells were immediately quantified using FCM (Beckman Coulter, Inc., Brea, CA, USA) and the results were analyzed using ModFit LT ${ }^{\mathrm{TM}} 4.1$ software (Verity Software House, Inc., Topsham, ME, USA).

WB. HCT116 and HT29 cells were homogenized in a lysate buffer (Beyotime Institute of Biotechnology, Shanghai, China), and protein concentrations were determined with a bicinchoninic acid kit (Beyotime). Protein extracts $(30 \mu \mathrm{g})$ were separated by $10 \%$ SDS-PAGE and transferred to polyvinylidene difluoride membranes (EMD Millipore, Billerica, MA, USA). The blots were blocked with 5\% non-fat milk for $1 \mathrm{~h}$ at room temperature, followed by incubation with primary antibodies overnight at $4^{\circ} \mathrm{C}$. The primary antibodies used were: Anti-GAPDH (1:2,000 dilution; cat. no. 10494-1-AP; Wuhan Sanying Biotechnology, Wuhan, China), anti-RANBP9 (1:1,000 dilution; cat. no. 17755-1-AP; Wuhan Sanying Biotechnology), anti-cyclin A2 (1:1,000 dilution; cat. no. 18202-1-AP; Wuhan Sanying Biotechnology) and anti-cyclin B1 (1:1,000 dilution; cat. no. 55004-1-AP; Wuhan Sanying Biotechnology). A horseradish peroxidase-conjugated anti-rabbit antibody as the secondary antibody (1:10,000; cat. no. KGAA35; Nanjing KeyGen Biotech Co., Ltd.) was incubated for $1 \mathrm{~h}$ at room temperature. The immunoreactive bands were visualized by the Tanon-4500 Gel Imaging system (Tanon Science and Technology Co., Ltd., Shanghai, China) using an enhanced chemiluminescence kit (Thermo Fisher Scientific, Inc.).

In vivo tumorigenicity assay. A total of 12 specific pathogen-free male BALB/c mice (4 weeks old; 14-18 g) were purchased from Shanghai SIPPR-Bk Lab Animal Co., Ltd. (Shanghai, China). All animals were housed under a temperature of $22 \pm 1^{\circ} \mathrm{C}$ and a relative humidity of $50 \pm 1 \%$ in a $12-\mathrm{h}$ light/dark cycle with free access to food and water. All studies were approved by the Shanghai Public Health Clinical Center Laboratory Animal Welfare and Ethics Committee (IRB no. 2018-A001-01; Shanghai, China). All mice were handled according to the National Institutes of Health Guidelines for the Care and Use of Laboratory Animals (16). Three animals per group (4 groups: The control and RANBP9-shRNA group for HCT116 cells or HT29 cells) were used in each experiment. Mice tissues were homogenized in a lysate buffer (Beyotime) and underwent WB. HCT116 or HT29 cells (expressing control-shRNA or RANBP9-shRNA) in $100 \mu \mathrm{l}$ PBS at $1 \times 10^{8}$ cells $/ \mathrm{ml}$ were inoculated into the armpit of the mice. The tumor volumes were measured weekly and calculated using the following formula: $\pi / 6 \mathrm{x}$ length $\mathrm{x}$ width ${ }^{2}$.

Statistical analysis. RANBP9 expression in human tissues was analyzed by Wilcoxon test. All data are expressed as the means \pm standard deviation of three independent experiments. Results were analyzed using a Student's t-test, or one-way analysis of variance followed by a least significant difference post hoc test, using the SPSS version 23 software (IBM Corp., 
Table I. Expression of RANBP9 in 75 colorectal cancer samples.

\begin{tabular}{lrrrrr}
\hline & \multicolumn{3}{c}{ RANBP9 immunostaining } & \\
\cline { 2 - 4 } Sample type & +++ & ++ & + & - & Positive rate $(\%)$ \\
\hline Cancer & 14 & 24 & 31 & 6 & 92.0 \\
Normal mucosa & 0 & 10 & 28 & 37 & 50.7 \\
\hline
\end{tabular}

${ }^{a}$ Wilcoxon test was used to determine statistical significance between the two groups. +++ , strongly positive;,++ moderately positive; + , slightly positive; -, negative. RANBP9, RAN binding protein 9.
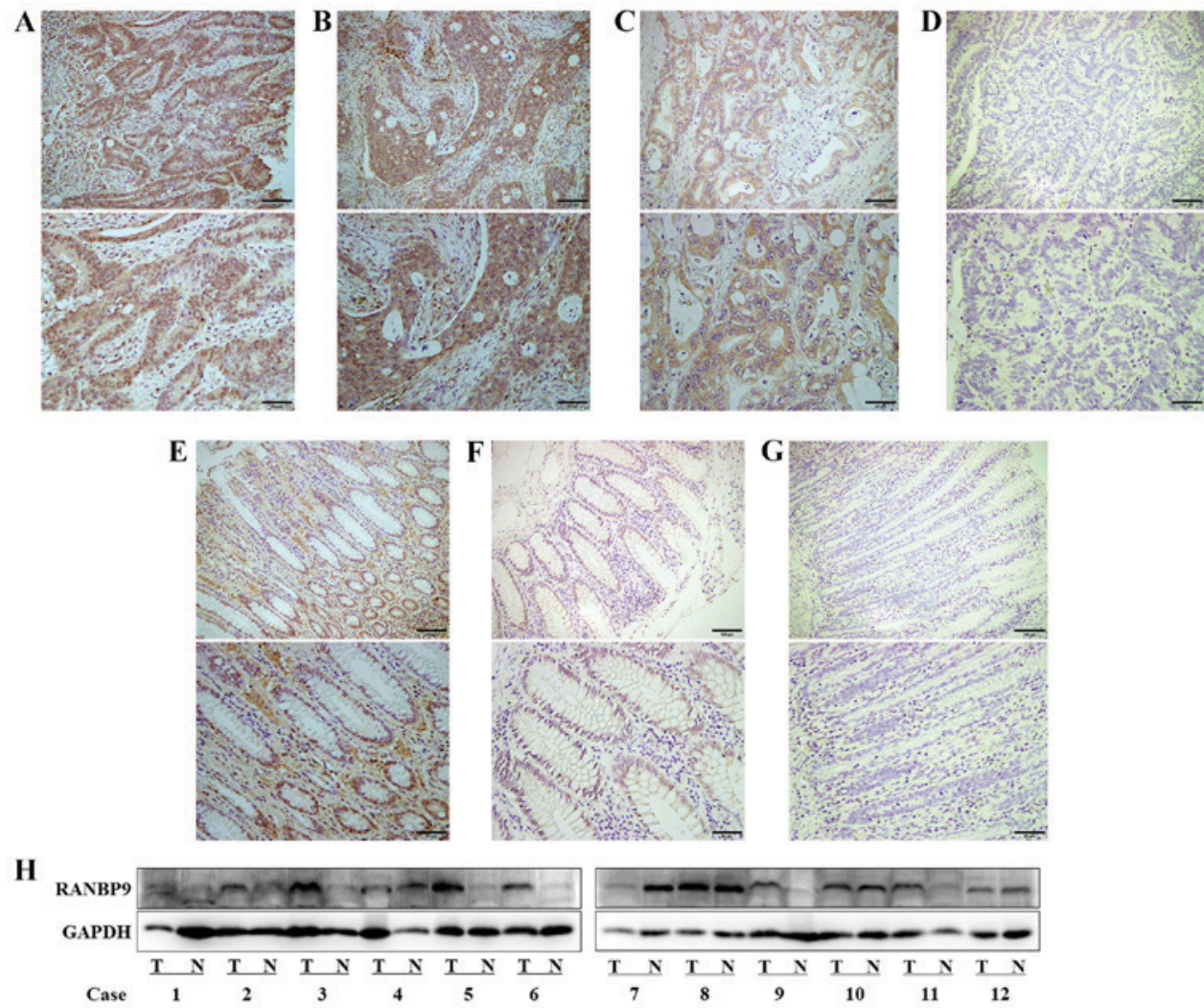

Figure 1. Expression of RANBP9 in paraffin-embedded and fresh colorectal cancer tissue samples. In tumor samples, (A) high-intensity RANBP9 expression was scored as '+++', (B) moderate-intensity RANBP9 expression as '++', (C) low-intensity RANBP9 expression as '+' and (D) the near absence of RANBP9 staining was scored as '-'. (E-G) Moderate, low and negative expression levels of RANBP9 in normal mucosa, respectively. (H) Expression levels of RANBP9 in 12 colorectal cancer samples as demonstrated by western blotting. Scale bars: Upper rows, $100 \mu$ m; lower rows, $50 \mu$ m. N, normal mucosa; RANBP9, RAN binding protein 9; $\mathrm{T}$, tumor.

Armonk, NY, USA). P<0.05 was considered to indicate a statistically significant difference.

\section{Results}

RANBP9 is overexpressed in human CRC. RANBP9 expression was detected in the paraffin-embedded CRC tissue samples using IHC (Fig. 1A-G) and in fresh tissue samples using WB (Fig. 1H). The results revealed that RANBP9 was overexpressed in CRC tissues compared with in adjacent normal tissues (two-sided Wilcoxon test, $\mathrm{P}<0.0001$; Table I). RANBP9 was detected in the nucleus and cytoplasm. These results indicated that high levels of RANBP9 were present in CRC. In the majority of fresh tissues, RANBP9 expression was higher in the tumor tissue than in the adjacent normal tissue. Additionally, the protein expression levels of RANBP9 in CRC cell lines were confirmed by WB (Fig. 2A). HCT116 and HT29 cells were selected for subsequent experiments as they are highly invasive CRC cell lines and exhibited high expression levels of RANBP9.

RANBP9 knockdown promotes proliferation of CRC cells. RANBP9 overexpression in CRC suggested that it may be associated with the biological behavior of CRC. RANBP9-shRNA and control-shRNA were successfully infected into HCT116 and HT29 cells using a lentiviral vector (Fig. 2B). HCT116 and HT29 cells were successfully transfected with RANBP9 plasmid or empty vector (Fig. 2C). A CCK-8 assay 
revealed that the number of viable cells at the last time point (72 $\mathrm{h}$ ) in the RANBP9-shRNA group was significantly higher compared with in the control group $(\mathrm{P}<0.01$; Fig. $3 \mathrm{~A}$ and $\mathrm{B})$. In addition, a colony formation assay was performed to determine tumorigenesis in vitro. Compared with in the control group, the number of HCT116 and HT29 cell colonies in the $R A N B P 9$-shRNA group was significantly higher $(\mathrm{P}<0.05$; Fig. $3 \mathrm{C}$ and $\mathrm{D})$. These results suggested that $R A N B P 9$-shRNA exhibited a positive effect on CRC cell proliferation.

RANBP9 overexpression hinders the proliferation of CRC cells. A CCK-8 assay revealed that the number of viable cells at the final timepoint $(72 \mathrm{~h}$ ) in the $R A N B P 9$ group was significantly lower compared with in the empty vector group ( $\mathrm{P}<0.01$; Fig. 4A and B). Similarly, in a colony formation assay, the number of HCT116 and HT29 cell colonies in the RANBP9 group was significantly lower compared with in the empty vector group $(\mathrm{P}<0.05$; Fig. $4 \mathrm{C}$ and $\mathrm{D})$. These data suggested an inhibitory role of RANBP9 in CRC cell proliferation.

RANBP9-shRNA promotes colon tumorigenesis in vivo. To examine the effects of RANBP9 knockdown on tumor growth in vivo, HCT116 and HT29 cells that stably expressed $R A N B P 9$-shRNA or control-shRNA were inoculated into the armpit of $\mathrm{BALB} / \mathrm{c}$ mice. All animals developed tumors within 1 week following inoculation. At 4 weeks post-inoculation, tumor sizes of the RANBP9-shRNA animals were larger than those of the control-shRNA group (Fig. 5A and B). The mean tumor volume after 4 weeks was $181.3 \pm 67.7 \mathrm{~mm}^{3}$ in mice inoculated with HCT116 cells that expressed RANBP9-shRNA compared with $33.7 \pm 8.5 \mathrm{~mm}^{3}$ in the control-shRNA group (Fig. 5C). The mean tumor volume of mice inoculated with HT29 cells transfected with RANBP9-shRNA was $925.3 \pm 427.3 \mathrm{~mm}^{3}$ compared with a volume of $184.3 \pm 48.5 \mathrm{~mm}^{3}$ in the control group (Fig. 5D). These data demonstrated the role of RANBP9-shRNA in promoting the growth of CRC cells in vivo. The efficiency of RANBP9-knockdown in lentivirus-infected HCT116 and HT29 cells was ensured according to the detection of RANBP9 expression level in transplanted tumors using WB (Fig. 5E and F).

RANBP9-shRNA facilitates the transition from $S$ phase in $C R C$ cells. Cell cycle profiling by FCM indicated that knockdown of RANBP9 expression resulted in a decreased number of cells in S phase in HCT116 and HT29 cells (Fig. 6A and B). However, no consistency was observed in the distribution between $\mathrm{G}_{1}$ and $\mathrm{G}_{2}$ stages for the two cell lines. These results suggested that $R A N B P 9$-shRNA promoted the proliferation of CRC cells indicated in the CCK-8 assay and the colony formation assay, by facilitating the transition from $\mathrm{S}$ phase. Additionally, cell cycle-associated protein expression levels were examined by WB following knockdown of RANBP9. RANBP9 knockdown yielded a variably increased protein level of cyclin A2 in the two cell lines (Fig. 6C and D). In addition, HT29 cells exhibited a lower expression of cyclin B1.

\section{Discussion}

In the present study, RANBP9 expression was significantly increased in tumor tissues compared with in paired normal
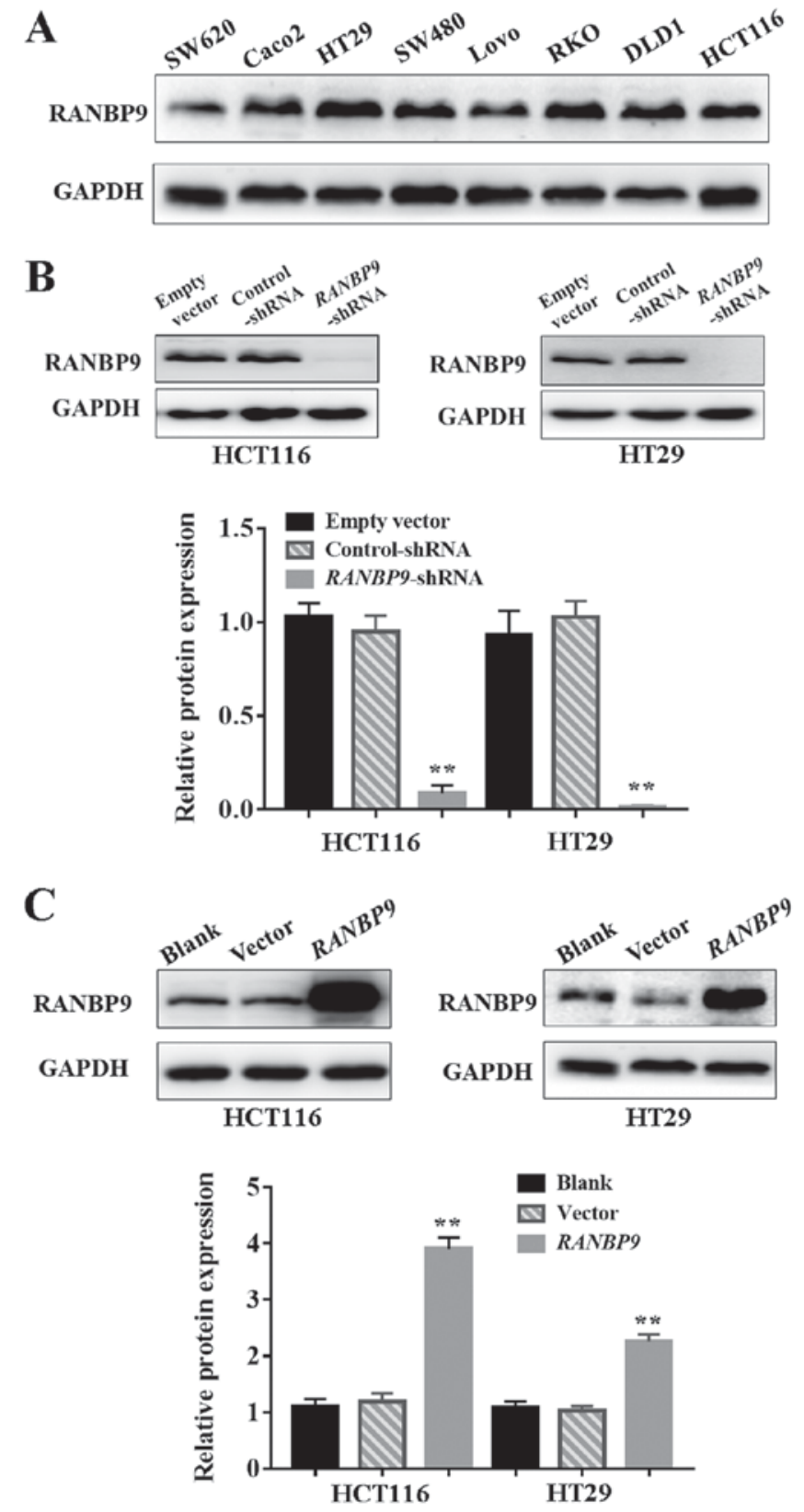

Figure 2. Analysis of RANBP9 protein expression following infection with control-shRNA and $R A N B P 9$-shRNA, or transfection with an empty vector and $R A N B P 9$ vector. (A) Western blot analysis of RANBP9 expression in eight colorectal cancer cell lines. (B) RANBP9 protein expression was significantly downregulated in $R A N B P 9$-shRNA lentivirus-infected HCT116 and HT29 cells compared with in cells infected with empty vector or control-shRNA lentivirus. (C) RANBP9 protein was significantly upregulated in $R A N B P 9$ vector-transfected cells compared with in blank cells and cells transfected with empty vector. Data are expressed as the means \pm standard deviation. ${ }^{* *} \mathrm{P}<0.01$, compared with control or vector cells. RANBP9, RAN binding protein 9; shRNA, short hairpin RNA.

mucosa tissues in patients with CRC, which indicated that RANBP9 may be associated with CRC. To further explore the function of RANBP9, its expression in several CRC cell lines was examined. The suppression of RANBP9 promoted proliferation and strengthened colony formation in CRC cells; accordingly, its overexpression led to suppressed proliferation and impaired colony formation. Additionally, shortening of the $\mathrm{S}$ phase was identified in two CRC cell lines. The present study revealed that RANBP9 may be 


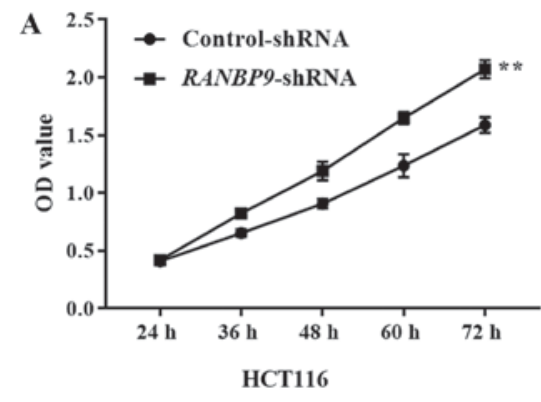

C
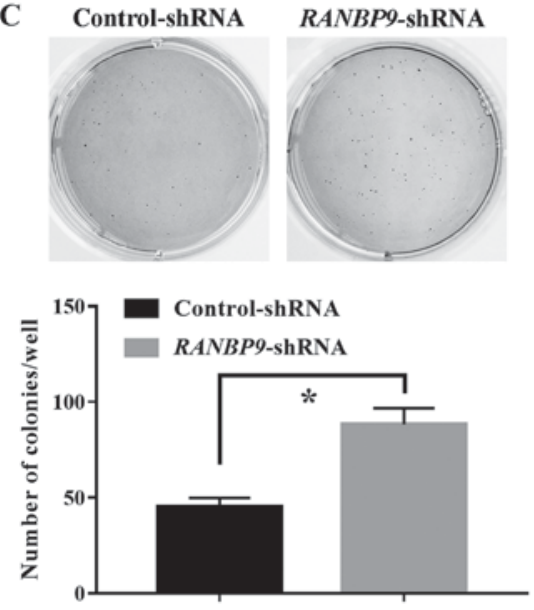

HCT116

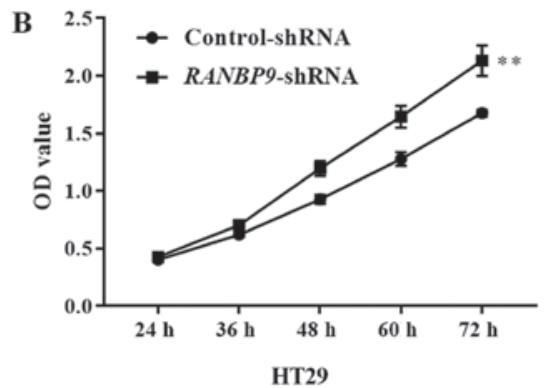

D
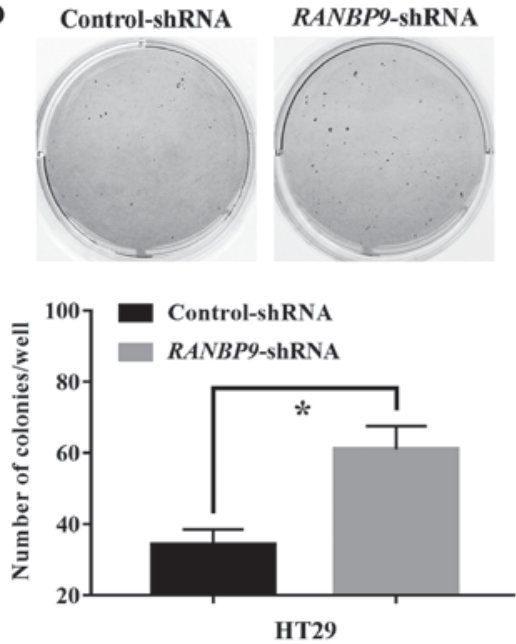

Figure 3. Effect of RANBP9 knockdown on proliferation and colony formation of colorectal cancer cells in vitro. Cell viability analysis of (A) HCT116 and (B) HT29 cells infected with control-shRNA or RANBP9-shRNA by Cell Counting kit- 8 assay measured at various time points over 3 days. Colony formation analysis of (C) HCT116 and (D) HT29 cells infected with control-shRNA or RANBP9-shRNA at 2 weeks following culture. Data are expressed as the means \pm standard deviation. ${ }^{*} \mathrm{P}<0.05,{ }^{* *} \mathrm{P}<0.01$. OD, optical density; RANBP9, RAN binding protein 9; shRNA, short hairpin RNA.
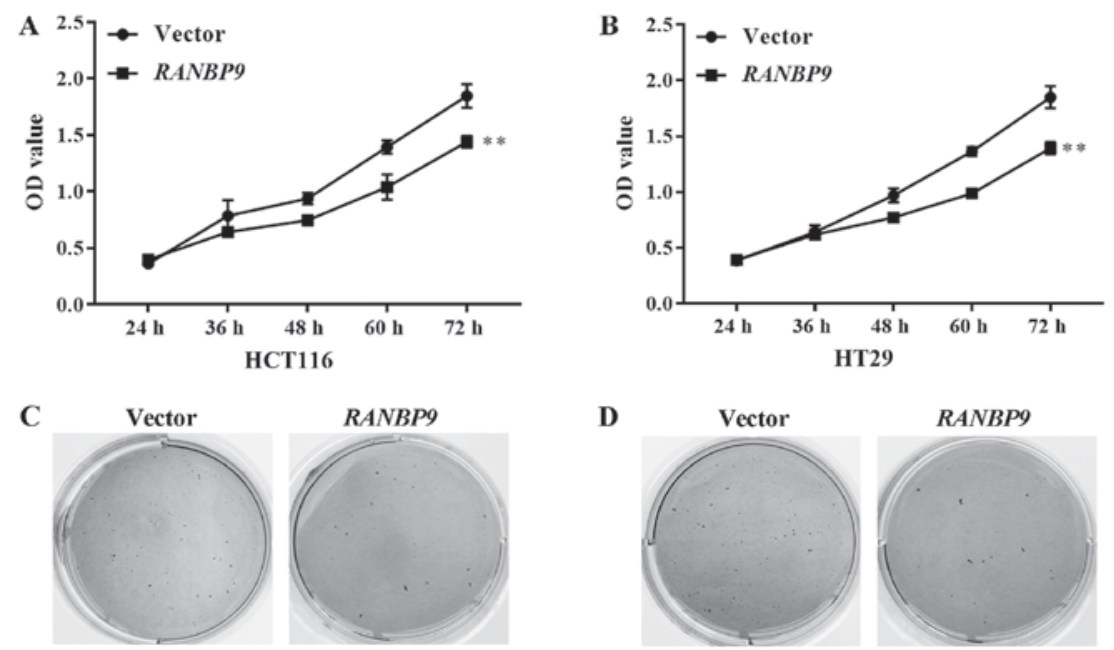

RANBP9

D
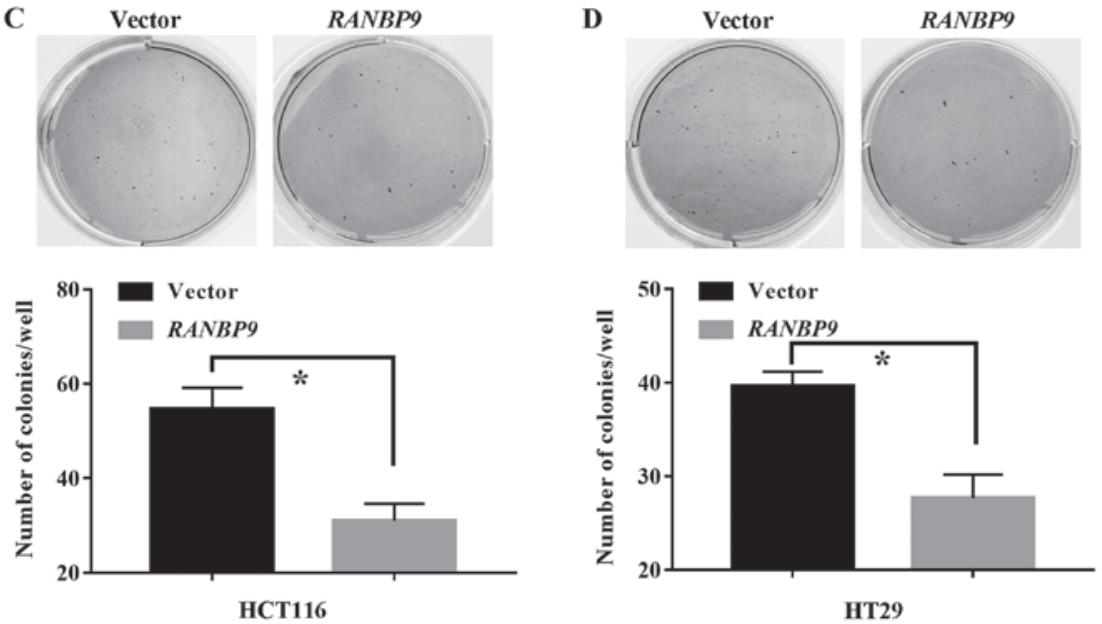

Figure 4. Effect of RANBP9 overexpression on proliferation and colony formation of colorectal cancer cells in vitro. Analysis of cell viability of (A) HCT116 and (B) HT29 cells transfected with empty vector or RANBP9 vector by Cell Counting kit- 8 assay measured at various time points over 3 days. Colony formation analysis of (C) HCT116 and (D) HT29 cells transfected with empty vector or $R A N B P 9$ vector at 2 weeks after culture. ${ }^{*} \mathrm{P}<0.05,{ }^{* * *} \mathrm{P}<0.01$. OD, optical density; RANBP9, RAN binding protein 9. 
A

RANBP9-shRNA

Control-shRNA

RANBP9-shRNA

Control-shRNA
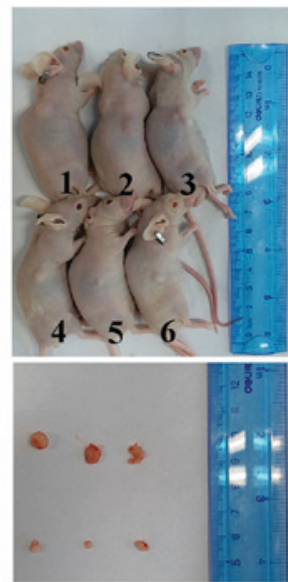

HCT116

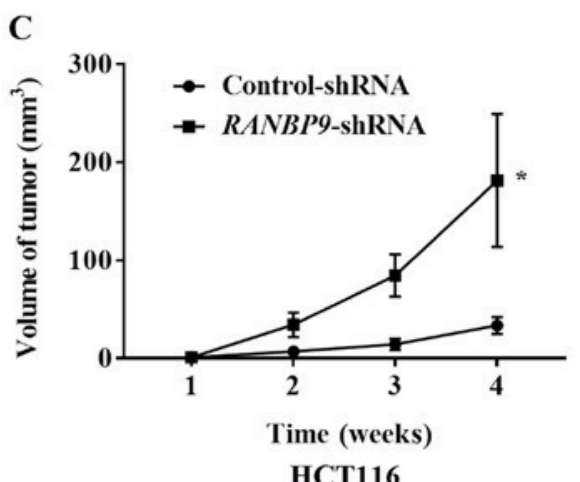

HCT116

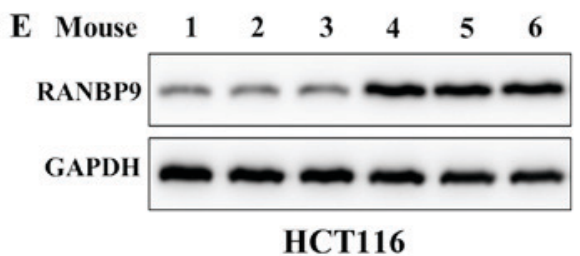

B

RANBP9-shRNA

Control-shRNA

RANBP9-shRNA

Control-shRNA
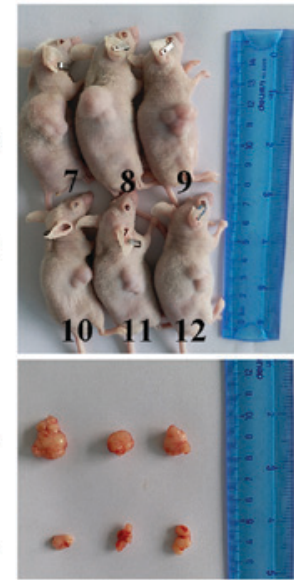

HT29
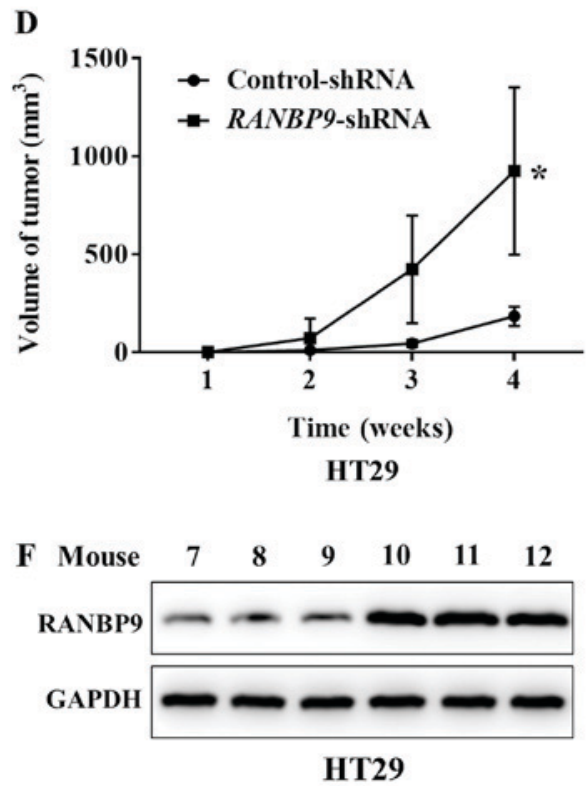

Figure 5. RANBP9 knockdown promotes colon tumorigenesis in vivo. (A and B) Representative images of BALB/c mice bearing tumors 4 weeks after inoculation with control shRNA or RANBP9-shRNA lentivirus-infected HCT116 and HT29 cells. (C and D) Average tumor volume at each time point. (E and F) Expression levels of RANBP9 in transplanted tumors were detected to ensure the efficiency of RANBP9 knockdown in lentivirus-infected HCT116 and HT29 cells. Lanes 1-12 corresponds to nude mice 1-12. " $\mathrm{P}<0.05$. RANBP9, RAN binding protein 9; shRNA, short hairpin RNA.

a potential anti-oncogene in CRC. These results were consistent with studies on osteosarcoma, lung and gastric cancer (10-13).

The present study demonstrated that RANBP9 exhibited an inhibitory role in CRC, yet it remains unclear why malignant cells overexpress RANBP9. Similar phenomena have been observed in osteosarcoma, lung and gastric cancer (10-13). Cancer cells are associated with different sets of oncogenes and tumor suppressor genes that are normally involved in stabilizing negative feedback loops. These genes do not act individually, but rather execute their cellular functions in cooperation with other genes, including oncogene mouse double minute 2 and tumor suppressor gene p53, which are overexpressed in hepatocellular cancer $(17,18)$, or Wnt signaling and conductin, which are upregulated in colorectal and liver tumors (19). Nevertheless, further studies should be conducted to elucidate the oncogene partner of $R A N B P 9$ in CRC.

The cell cycle is a complex system, which includes phases that are comprehensively regulated by cyclin-dependent kinases (CDKs), cyclins, catalytic partners and CDK inhibitors, directly affecting the proliferation and tissue differentiation of mammalian cells $(20,21)$. In numerous diseases, the mechanism of normal cell division is constantly altered, particularly in several types of cancer. Cyclins and CDKs, including cyclin D, cyclin E, CDK4 and CDK6, have been classified as proto-oncogenes, while CDK inhibitors, including p16 and p21, are regarded as tumor suppressors; all of these are associated with the occurrence or prognosis of CRC $(20,22,23)$. Therefore, cell cycle-targeted therapy is a possible way to control the disease. For example, palbociclib, which is an orally administered and specific CDK4/6 inhibitor, can prominently inhibit tumor growth in mice bearing colon cancer (24). Flavopiridol, which inhibits CDK1, CDK2 and CDK4, has also been widely studied in gastrointestinal cancer $(25,26)$. There are additional cell cycle inhibitors in different stages of development; nonetheless, to the best of our knowledge, there are still no encouraging reports. In the present study, RANBP9-shRNA promoted S phase transition 

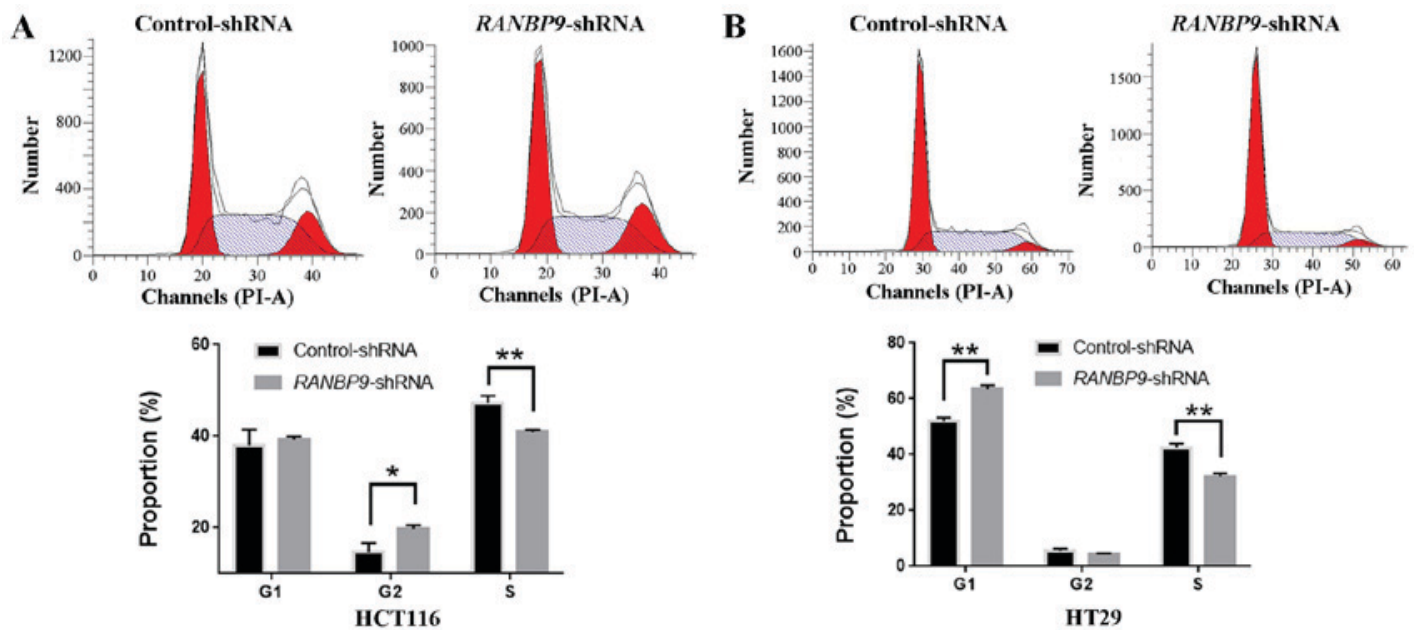

C

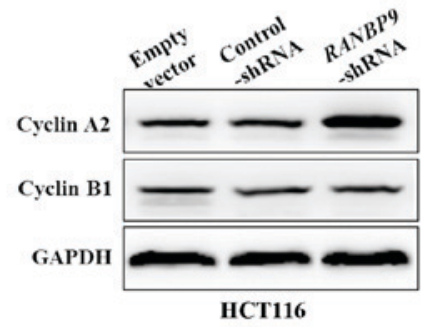

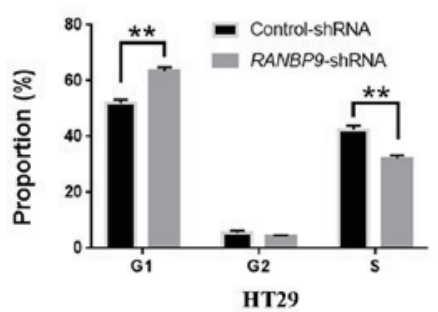

D

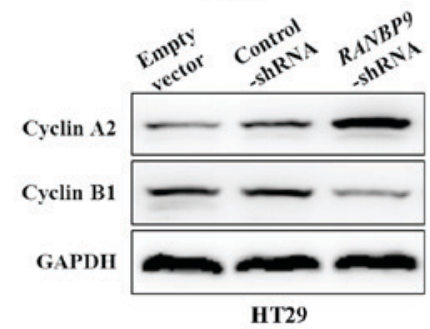

Figure 6. RANBP9 knockdown decreases the proportion of colorectal cancer cells in S phase. (A and B) Effect of RANBP9 knockdown on the cell cycle progression of HCT116 and HT29 cells analyzed by flow cytometry. The percentages of cells in the $\mathrm{G}_{1}, \mathrm{G}_{2}$ and $\mathrm{S}$ phases are shown. (C and D) Representative western blotting images of cyclin A2 and cyclin B1 in HCT116 and HT29 cells infected with empty vector, control-shRNA or RANBP9-shRNA lentivirus. GAPDH was used as a loading control. ${ }^{*} \mathrm{P}<0.05,{ }^{* *} \mathrm{P}<0.01$. RANBP9, RAN binding protein 9; shRNA, short hairpin RNA.

in HCT116 and HT29 cells, although there were no consistent results for the $G_{1}$ or $G_{2}$ phases. CDK/Cyclin A2 complexes have been reported to phosphorylate proteins, including pocket proteins $\mathrm{Rb}, \mathrm{p} 107, \mathrm{p} 130$, and proteins involved in DNA synthesis, thereby driving $\mathrm{S}$ phase progression $(27,28)$. In the present study, it was indicated that RANBP9-knockdown increased protein level of cyclin A2 in the two cell lines. In addition, RANBP9-shRNA promotes cell proliferation by CCK8 assay and colony formation assay. These suggest that $R A N B P 9$-shRNA may facilitate $\mathrm{S}$ phase transition rather than induce $\mathrm{G}_{1}$ or $\mathrm{G}_{2} / \mathrm{M}$ arrest. Further studies are required in order to prove that RANBP9 overexpression induces cell cycle arrest at $\mathrm{S}$ phase.

At present, eukaryotic cyclins, including cyclin A-H, which are synthesized and degraded in different phases of the cell cycle, are fluctuant and periodic-phase specific (29). Cyclin A serves a role in the later stages of the $S$ phase; cyclin $B$ is mainly associated with the $G_{2} / M$ phase; cyclin $D$ and cyclin $E$ serve a key regulatory role in the $\mathrm{G}_{1} / \mathrm{S}$ phase; and cyclin $F$ and cyclin $G$ are associated with the late $S$ and $G_{2} / M$ phase $(29,30)$. The results of the present study revealed that there may be an association between cyclin A and RANBP9 knockdown in CRC cells. However, it may be a phenomenon rather than a direct regulatory association, which requires further examination.

There are limitations in the present study. Firstly, the clinical sample size was small and obtained at a single center, which may lead to selective bias. In addition, RANBP9 expression in fresh normal intestinal epithelium in certain cases was higher compared with in matched tumor tissues, which remains unexplained. RANBP9 has numerous characteristics as a scaffold protein, including a protein interaction sequence, cytoskeleton binding domain and multiple classical anchor loci for signal transduction molecules $(4,31)$; it can interact with SRC proto-oncogene non-receptor kinase, growth factor receptor bound protein 2 and hepatocyte grow th factor receptor in the mitogen-activated protein kinase and extracellular signal-regulated kinase signaling pathways (4,32-34). The downstream mechanism of RANBP9 in CRC cells should be examined in subsequent experiments.

In conclusion, RANBP9 is a molecule associated with the complex cell cycle regulation network. The present study revealed that RANBP9 contributed to tumor suppression in CRC. Exploring the underlying mechanisms of RANBP9 activity may lead to its use in the treatment of patients with CRC.

\section{Acknowledgements}

Not applicable.

\section{Funding}

The study was funded by the Health and Family Planning Commission of Jinshan District, Shanghai, China (grant no. JSKJ-KTMS-2017-01).

\section{Availability of data and materials}

All data generated or analyzed during this study are included in this published article. 


\section{Authors' contributions}

CQ and GW designed the study and analyzed the data. CQ and QZ performed the experiments. All authors read and approved the final manuscript.

\section{Ethics approval and consent to participate}

All patients were required to provide written informed consent prior to their inclusion. The study was approved by the Ethics Committee of Jinshan Hospital, Fudan University. All animal studies were approved by the Shanghai Public Health Clinical Center Laboratory Animal Welfare and Ethics Committee (IRB no. 2018-A001-01).

\section{Patient consent for publication}

All patients provided written informed consent for the publication of their data.

\section{Competing interests}

The authors declare that they have no competing interests.

\section{References}

1. Miller KD, Siegel RL, Lin CC, Mariotto AB, Kramer JL, Rowland JH, Stein KD, Alteri R and Jemal A: Cancer treatment and survivorship statistics, 2016. CA Cancer J Clin 66: 271-289, 2016.

2. Fearon ER and Vogelstein B: A genetic model for colorectal tumorigenesis. Cell 61: 759-767, 1990.

3. Yokoyama N, Hayashi N, Seki T, Panté N, Ohba T, Nishii K, Kuma K, Hayashida T, Miyata T and Aebi U: A giant nucleopore protein that binds Ran/TC4. Nature 376: 184-188, 1995.

4. Murrin LC and Talbot JN: RanBPM, a scaffolding protein in the immune and nervous systems. J Neuroimmune Pharmacol 2: 290-295, 2007.

5. Salemi LM, Loureiro SO and Schild-Poulter C: Characterization of RanBPM molecular determinants that control its subcellular localization. PLoS One 10: e0117655, 2015.

6. Wang H, Lewsadder M, Dorn E, Xu S and Lakshmana MK RanBP9 overexpression reduces dendritic arbor and spine density. Neuroscience 265: 253-262, 2014.

7. Wang R, Palavicini JP, Wang H, Maiti P, Bianchi E, Xu S, Lloyd BN, Dawson-Scully K, Kang DE and Lakshmana MK: RanBP9 overexpression accelerates loss of dendritic spines in a mouse model of Alzheimer's disease. Neurobiol Dis 69: 169-179, 2014.

8. Valiyaveettil M, Bentley AA, Gursahaney P, Hussien R, Chakravarti R, Kureishy N, Prag S and Adams JC: Novel role of the muskelin-RanBP9 complex as a nucleocytoplasmic mediator of cell morphology regulation. J Cell Biol 182: 727-739, 2008.

9. Rex EB, Rankin ML, Yang Y, Lu Q, Gerfen CR, Jose PA and Sibley DR: Identification of RanBP 9/10 as interacting partners for protein kinase $\mathrm{C}(\mathrm{PKC})$ gamma/delta and the D1 dopamine receptor: Regulation of PKC-mediated receptor phosphorylation. Mol Pharmacol 78: 69-80, 2010.

10. Zhao Z, Cheng S, Zabkiewicz C, Chen J, Zhang L, Ye L and Jiang WG: Reduced expression of ranBPM is associated with poorer survival from lung cancer and increased proliferation and invasion of lung cancer cells in vitro. Anticancer Res 37 4389-4397, 2017.

11. Shao S, Sun PH, Satherley LK, Gao X, Ji KE, Feng YI, Jia Y, Ji J, Jiang WG and Ye L: Reduced ranBPM expression is associated with distant metastasis in gastric cancer and chemoresistance. Anticancer Res 36: 1295-1303, 2016

12. Dai H, Lv YF, Yan GN, Meng G, Zhang X and Guo QN RanBP9/TSSC3 complex cooperates to suppress anoikis resistance and metastasis via inhibiting Src-mediated Akt signaling in osteosarcoma. Cell Death Dis 7: e2572, 2016.
13. Zhu LL, Wang CH, Yang HP and Shu WH: Expression of cartilage antitumor component RanBP9 in osteosarcoma. J Biol Regul Homeost Agents 30: 103-110, 2016.

14. Emberley ED, Gietz RD, Campbell JD, HayGlass KT, Murphy LC and Watson PH: RanBPM interacts with psoriasin in vitro and their expression correlates with specific clinical features in vivo in breast cancer. BMC Cancer 2: 28, 2002.

15. Qin C, Ren L, Ji M, Lv S, Wei Y, Zhu D, Lin Q, Xu P, Chang W and $\mathrm{Xu} \mathrm{J}$ : CDKL1 promotes tumor proliferation and invasion in colorectal cancer. Onco Targets Ther 10: 1613-1624, 2017.

16. Wang H, Yang R, Zhong L, Zhu XY, Ma PP, Yang XQ, Jiang KL and Liu BZ: Location of NLS-RARalpha protein in NB4 cell and nude mice. Oncol Lett 13: 2045-2052, 2017.

17. Meng X, Franklin DA, Dong J and Zhang Y: MDM2-p53 pathway in hepatocellular carcinoma. Cancer Res 74: 7161-7167, 2014.

18. Zhang MF, Zhang ZY, Fu J, Yang YF and Yun JP: Correlation between expression of $\mathrm{p} 53, \mathrm{p} 21 / \mathrm{WAF} 1$, and MDM2 proteins and their prognostic significance in primary hepatocellular carcinoma. J Transl Med 7: 110, 2009.

19. Lustig B, Jerchow B, Sachs M, Weiler S, Pietsch T, Karsten U, van de Wetering M, Clevers H, Schlag PM, Birchmeier W and Behrens J: Negative feedback loop of Wnt signaling through upregulation of conductin/axin2 in colorectal and liver tumors. Mol Cell Biol 22: 1184-1193, 2002.

20. Malumbres M: Cyclin-dependent kinases. Genome Biol 15: 122 , 2014.

21. Morgan DO: Cyclin-dependent kinases: Engines, clocks, and microprocessors. Annu Rev Cell Dev Biol 13: 261-291, 1997.

22. Ogino S, Nosho K, Irahara N, Shima K, Baba Y, Toyoda S, Chen L, Giovannucci EL, Meyerhardt JA and Fuchs CS: A cohort study of cyclin D1 expression and prognosis in 602 colon cancer cases. Clin Cancer Res 15: 4431-4438, 2009.

23. Zhao P, Hu YC and Talbot IC: Expressing patterns of p16 and CDK4 correlated to prognosis in colorectal carcinoma. World J Gastroenterol 9: 2202-2206, 2003.

24. Fry DW, Harvey PJ, Keller PR, Elliott WL, Meade M, Trachet E, Albassam M, Zheng X, Leopold WR, Pryer NK and Toogood PL: Specific inhibition of cyclin-dependent kinase 4/6 by PD 0332991 and associated antitumor activity in human tumor xenografts. Mol Cancer Ther 3: 1427-1438, 2004.

25. Aklilu M, Kindler HL, Donehower RC, Mani S and Vokes EE: Phase II study of flavopiridol in patients with advanced colorectal cancer. Ann Oncol 14: 1270-1273, 2003.

26. Motwani M, Rizzo C, Sirotnak F, She Y and Schwartz GK Flavopiridol enhances the effect of docetaxel in vitro and in vivo in human gastric cancer cells. Mol Cancer Ther 2: 549-555, 2003.

27. Yam CH, Fung TK and Poon RY: Cyclin A in cell cycle control and cancer. Cell Mol Life Sci 59: 1317-1326, 2002.

28. Zindy F, Lamas E, Chenivesse X, Sobczak J, Wang J, Fesquet D, Henglein B and Bréchot C: Cyclin A is required in S phase in normal epithelial cells. Biochem Biophys Res Commun 182: 1144-1154, 1992

29. Bloom J and Cross FR: Multiple levels of cyclin specificity in cell-cycle control. Nat Rev Mol Cell Biol 8: 149-160, 2007.

30. Malumbres M and Barbacid M: Cell cycle, CDKs and cancer: A changing paradigm. Nat Rev Cancer 9: 153-166, 2009.

31. Lakshmana MK, Chung JY, Wickramarachchi S, Tak E, Bianchi E, Koo EH and Kang DE: A fragment of the scaffolding protein RanBP9 is increased in Alzheimer's disease brains and strongly potentiates amyloid-beta peptide generation. FASEB J 24: 119-127, 2010.

32. Brannetti B and Helmer-Citterich M: iSPOT: A web tool to infer the interaction specificity of families of protein modules. Nucleic Acids Res 31: 3709-3711, 2003.

33. Wang D, Li Z, Messing EM and Wu G: The SPRY domain-containing SOCS box protein 1 (SSB-1) interacts with MET and enhances the hepatocyte growth factor-induced Erk-Elk-1-serum response element pathway. J Biol Chem 280: 16393-16401, 2005.

34. Woo JA, Roh SE, Lakshmana MK and Kang DE: Pivotal role of RanBP9 in integrin-dependent focal adhesion signaling and assembly. FASEB J 26: 1672-1681, 2012.

This work is licensed under a Creative Commons Attribution-NonCommercial-NoDerivatives 4.0 International (CC BY-NC-ND 4.0) License. 